\title{
LA VOLATIDAD DEL PRECIO DEL PETRÓleO: SUS EFECTOS EN LA ECONOMÍA ECUATORIANA EN LA ÚLTIMA DÉCADA
}

\section{THE VOLATITY OF PETROLEUM PRICE: ITS EFFECTS ON THE ECUADORIAN ECONOMY IN THE LAST DECADE}

Claudia Beatriz Jurado Parra ${ }^{1}$ Holger Fabrizzio Bejarano Copo ${ }^{2}$ Virgilio Eduardo Salcedo Muñoz ${ }^{3}$

Marco Sánchez Maldonado 4

1. Economista con Mención en Gestión Empresarial (Ecuador). E-mail: c-b-j-p@hotmail.com

2. Economista. Docente titular Universidad Técnica de Machala, UTMACH (Ecuador).

E-mail: hbejarano@utmachala.edu.ec

3. Economista. Docente titular Universidad Técnica de Machala, UTMACH (Ecuador).

E-mail:vsalcedo@utmachala.edu.ec

4. Ingeniero. Docente Titular Universidad Técnico de Machala, UTMACH (Ecuador). E-mail: msanchez@utmachala.edu.ec

\section{Citación sugerida:}

Jurado Parra, C.B., Bejarano Copo, H.F., Salcedo Muñoz, V.E. y Sánchez Maldonado, M. (2017). La volatidad del precio del petróleo: sus efectos en la economía ecuatoriana en la última década. $3 C$ Empresa: investigación y pensamiento crítico, 6(2), 33-45. DOI: $<$ http://dx.doi.org/10.17993/3cemp.2017.060230.33-45/>. 


\section{RESUMEN}

El mercado energético a nivel mundial ha presentado cambios repentinos en el precio internacional del barril de petróleo, generando de manera imprevista graves consecuencias económicas y sociales. Este suceso ha afectado a la economía ecuatoriana convirtiéndose para el país en unos de los temas fundamentales del 2016, debido al impacto directo que esta crisis tiene en los ingresos fiscales, por tal motivo, el presente trabajo de investigación describe de forma concisa los efectos que tiene el precio internacional del barril de petróleo en las principales variables macroeconómicas del periodo 2000-2015. Para el alcance de dicho objetivo se estudia la relación funcional del precio del petróleo con la Balanza Comercial, PIB, y el Presupuesto General del Estado. Se utilizó la metodología de regresión lineal simple en conjunto con el programa econométrico STATA12.0. Los resultados indican que el precio del petróleo tiene una estrecha relación con las variables objeto de estudio.

\section{ABSTRACT}

The energy market worldwide has presented sudden changes in the international price of a barrel of oil, generating unexpectedly severe economic and social consequences. This event has affected the Ecuadorian economy turning the country into one of the key issues of 2016 due the direct impact of this crisis on tax revenue, for this reason, this research paper concisely describes the effects of the international price of a barrel of oil in the main macroeconomic variables for the period 2000-2015. To reach this objective the functional relationship of oil price with the trade balance, GDP and the General State Budget is studied. The simple linear regression methodology in conjunction with the STATA12.0 econometric program was used. The results indicate that the oil price has a close relationship with the variables under study.

\section{PALABRAS CLAVE}

Crisis financiera, Recesión, Precio del petróleo, Incidencia, Volatilidad.

\section{KEY WORDS}

Financial crisis, Recession, Oil price, Incidence, Volatility. 


\section{INTRODUCCIÓN}

El petróleo, recurso natural extraído de rocas sedimentarias, sin lugar a dudas es considerado la fuente de energía más importante en las últimas décadas incidiendo en la vida política, social y económica mundial originando millonarios ingresos y fuentes de trabajo.

Durante la nueva era del siglo XXI se han hecho presente un sin número de eventos a raíz de la caída histórica del precio del petróleo, el cual es sensible a eventos de carácter económicos como extraeconómicos, generando gran impacto en los países netamente dependiente de sus rentas petroleras como los miembros de la OPEP.

Actualmente, según la Agencia Internacional de Energía (2012) el consumo mundial de petróleo alcanza los 93 millones de barriles al día, pero la oferta está alrededor de 97 millones de barriles debido a nuevas tecnologías (fracking) adoptadas por EEUU (pionero), esa sobreproducción es la que ha incidido en el precio. "Se prevé que Estados Unidos se convierta en el mayor productor mundial de petróleo hacia el 2020 (adelantando a Arabia Saudí hasta mediados de la década del 2020)".

El petróleo tiene "dimensiones oceánicas" por cuanto de él se obtienen más de 3.000 derivados, no existiendo ningún otro elemento, materia prima o energético en el planeta tierra donde se puedan obtener tantos productos y derivados impactando al crecimiento económico, Vargas (2008). La necesidad de Ecuador por importar estos derivados del petróleo, ante el fracaso de la industrialización del sector, ha hecho que una parte importante de sus rentas petroleras fluya al exterior (Mateo y García, 2014).

Los impactos de la crisis petrolera se sienten con creciente fuerza por todos los países de América Latina y no menos para Ecuador, por ser un país productor y exportador de petróleo que en su momento supo beneficiarse económicamente por los altos incrementos en las rentas petroleras. Debido al elevado precio del barril que llegó a su máximo nivel en junio de 2008 con un precio de USD \$121 por barril, desde ese momento los precios se desplomaron viviendo un escenario económico diferente con un precio por debajo de los USD \$50 a comienzos de año 2016.

Ante la caída abrupta del precio del barril convirtiéndose en una amenaza para la estabilidad del país, se genera la gran incógnita ¿Cómo se ve afectado económicamente el Ecuador?, es de interés analizar los efectos que ha tenido el precio del crudo en las principales variables económicas (PIB, Balanza Comercial y el precio anual promedio del barril de petróleo) durante los últimos 15 años. Para ello se realiza un análisis econométrico de cointegración donde se pueden observar las relaciones evidentes de los efectos de las variaciones del precio del barril de petróleo en el resto de las variables analizadas mediante el programa econométrico Stata12.0.

El análisis que se muestra corresponde desde el año 2001, año que está marcado por la dolarización y en donde probablemente existieron algunas otras variables que impactaron de manera negativa la excesiva volatilidad del precio del crudo, pero que para efectos de esta investigación no serán tomados en cuenta por estar fuera del objetivo central. 
La cointegración de las variables se ha aplicado de la siguiente manera: Primero se ha realizado un análisis bivariante de cada una de ellas y después el análisis de cointegración de la relación del precio del petróleo con el Presupuesto General del Estado. Los resultados obtenidos por el programa econométrico STATA12.0 nos muestran claramente que existen indicios evidentes de los efectos contraproducentes de los shocks petroleros en la economía ecuatoriana.

Esta investigación fue realizada con el refuerzo de la información de diferentes medios del país y del mundo para articular la serie procesos relacionados con dicha crisis. Estos son: Banco Central del Ecuador (BCE), Sistema Nacional de información (SIN), Banco Mundial (B.M), Fondo Monetario Internacional (FMI) y la Agencia Internacional de Energía.

\section{CARACTERISTICAS GENERALES DEL PETRÓLEO Y SU INCIDENCIA EN EL MUNDO}

En un contexto global, el petróleo es la materia prima más influyente en el mundo, debido a su participación en las crecientes necesidades energéticas, crecimiento económico y desarrollo social. Según Hernández (2015) la energía puede ser, y ha sido un instrumento de dominación, y alrededor de ella se construyeron los más poderosos imperios económicos y políticos.

Al mismo tiempo, las necesidades energéticas a nivel mundial son satisfechas en un $90 \%$ por combustibles fósiles, siendo el petróleo el más trascendental. Es importante observar los datos de la Corporación de Reservas de Productos Petrolíferos (CORES, 2016), que indica que la demanda de petróleo aumentó en el 2015 un 2,6\% respecto al año 2014.

Esto debido que durante el 2015 se incrementaron las importaciones de todas las zonas geográficas, destacando África y Oriente Medio, que ascendieron a 15,5\% y 13,0\%, respectivamente. Por otro lado, en los últimos años países como Nigeria, México y Arabia Saudí han sido los principales países suministradores de crudo a España.

De acuerdo a las predicciones de los geólogos más importantes a nivel mundial, este recurso natural alcanzará su nivel pico en la curva de producción a mediados del presente siglo, donde el $50 \%$ de las reservas habrán sido agotadas, desde ese punto el precio del barril seguirá creciendo de manera rápida afectando fuertemente a los países industrializados y a las economías emergentes (López \& Hilario, 2008).

\subsection{EL PRECIO DEL PETRÓleO Y CAUSAS DE SU RECIENTE VOLATIDAD}

Tras una época marcada por precios superiores a USD \$100 el barril de petróleo, para mediados del 2014, el mundo petrolífero se enfrenta a una incertidumbre sin precedentes, debido, a que esta materia prima presenta una fuerte reducción en el precio desde 
septiembre del 2015, empañando las perspectivas de crecimiento de los países exportadores de este recurso natural.

Esto se debe a que el descenso del precio del barril genera presión e incertidumbre en los ingresos fiscales de las naciones exportadoras, y que su presupuesto general del estado depende en gran medida en estos ingresos; al mismo tiempo los países importadores se están beneficiando plenamente de este abaratamiento debido al bajo costo comercial de la energía. Este impactante descenso de acuerdo a lo que observa el Fondo monetario Internacional (2016), fue provocado por el exceso de la sobre oferta que está estrechamente ligada a factores geopolíticos y tecnológicos, sin importar su afectación a países miembros y no miembros de la OPEP (Arroyo \& Cossío, 2015).

Por ejemplo, los estudios de Barrañón (2008) y Lorca (2015) deducen que las crisis petrolera tanto para México como España, fue generada por las limitaciones en el suministro de petróleo a nivel mundial por parte de la OPEP, originando grandes crisis en ambos Países: Para México debido a fuertes inversiones en el sector petrolero, y el descenso de los precios; y para España por ser un país Importador y dependiente de esta materia prima.

Existen múltiples estudios a nivel mundial, que explican la incidencia del precio del petróleo en el crecimiento económico de un país, ya sea exportador o importador. A continuación, en la tabla 1 se observan algunas de estas investigaciones que comprueban los impactos del shock petrolero en las diferentes variables económicas.

Tabla 1: Estudios del impacto del precio del petróleo en el crecimiento económico.

\begin{tabular}{|c|c|c|c|c|c|c|}
\hline $\begin{array}{l}\text { País origen } \\
\text { estudio }\end{array}$ & Autores & & Año & Tema & $\begin{array}{l}\text { Periodo de } \\
\text { estudio }\end{array}$ & variables \\
\hline Colombia & $\begin{array}{l}\text { González y } \\
\text { Hernández }\end{array}$ & & (2016) & $\begin{array}{l}\text { Impactos indirectos de } \\
\text { los precios del } \\
\text { petróleo }\end{array}$ & $2000-2013$ & $\begin{array}{l}\text { Precio petróleo, PIB, } \\
\text { Balanza comercial, } \\
\text { ITI, consumo } \\
\text { privado, IED, Balance } \\
\text { fiscal }\end{array}$ \\
\hline $\begin{array}{l}\text { Estados } \\
\text { Unidos }\end{array}$ & Hamilton & & (2009) & $\begin{array}{lc}\text { Causas } & y \\
\text { consecuencias de la } \\
\text { crisis del petróleo }\end{array}$ & $2007-2008$ & Producción, precio \\
\hline Rusia & Ito. $\mathrm{K}$ & & (2010) & $\begin{array}{l}\text { El impacto de la } \\
\text { volatilidad de los } \\
\text { precios del petróleo en } \\
\text { la actividad } \\
\text { macroeconómica }\end{array}$ & 1994-2009 & $\begin{array}{l}\text { Tipo de cambio, PIB, } \\
\text { Inflación, precio del } \\
\text { petróleo }\end{array}$ \\
\hline $\begin{array}{l}\text { Estados } \\
\text { Unidos }\end{array}$ & Kilian, L & & (2008) & $\begin{array}{l}\text { Los efectos } \\
\text { económicos de los } \\
\text { choques de los precios } \\
\text { de la energía }\end{array}$ & $\begin{array}{l}1970 \text { a Julio } \\
2006\end{array}$ & $\begin{array}{l}\text { Precio barril, PIB, } \\
\text { Inflación }\end{array}$ \\
\hline Ecuador & $\begin{array}{l}\text { Mateo } \\
\text { García }\end{array}$ & $y$ & 2014 & Petróleo en el Ecuador & $2000-2010$ & $\begin{array}{l}\text { Producción, Ingreso } \\
\text { fiscal, Inserción } \\
\text { externa }\end{array}$ \\
\hline $\begin{array}{l}\text { República } \\
\text { Dominicana }\end{array}$ & $\begin{array}{l}\text { Marte } \\
\text { Villanueva }\end{array}$ & $y$ & (2007) & $\begin{array}{l}\text { Precios internacionales } \\
\text { del petróleo }\end{array}$ & 2007 & Precio petróleo, PIB \\
\hline
\end{tabular}

Fuente: elaboración propia. 
Tal como se observa en la tabla anterior, varios estudios tanto en economías desarrolladas, como en vía de desarrollo, indican que la volatilidad del precio del petróleo, incide considerablemente en las variables que afectan el bienestar y crecimiento de los estados. Los autores Blanchard \& Gali (2007), en su estudio "los efectos macroeconómicos de las crisis del petróleo" hacen hincapié que la variabilidad del precio del petróleo cada vez es menos impactante en las economías desarrolladas, llegando a la conclusión de su investigación que los efectos de la crisis del petróleo han cambiado con el tiempo, con efectos cada vez más pequeños sobre los precios y los salarios, así como en la producción y el empleo.

\subsection{EL PETRÓLEO Y LA ECONOMÍA ECUATORIANA}

En América Latina, Ecuador es una de las economías más vulnerables frente a los comportamientos desfavorables de los precios del barril de petróleo determinando sus perspectivas económicas con ingresos petroleros superiores a 11 millones de dólares anuales.

Ecuador exporta alrededor del $70 \%$ crudo oriente y $30 \%$ de crudo napo, y a lo largo de la historia petrolera del país siempre se ha exportado el crudo Oriente y Napo por debajo del precio de referencia WTI. Se debe esto a que la calidad del crudo se mide a través de los índices de gravedad API, y contenido de azufre; por lo tanto, mientras más alto sea el contenido de azufre el precio de cotización es menor. WTI es un crudo liviano (40o API) fácil de refinar, el crudo Oriente es pesado (24 API-intermedio) y el crudo Napo (18 y $21^{\circ}$ APIpesado y agrio) esto hace q sea más complicada su refinación y evidencia una menor calidad. El crudo Napo y Oriente se venden por separado y a diferentes precios.

Es importante recalcar que hay un diferencial entre el precio internacional WTI (West Texas Intermédiate- tipo de crudo producido en USA, que sirve como referente a nivel mundial) y el precio de crudo ecuatoriano debido a que al ser de menor calidad es cotizado a un valor inferior. A mayor diferencial, menor es el precio que recibe el país por cada barril de petróleo exportado.

Los ingresos petroleros, durante décadas se han constituido en la principal fuente de ingresos estatales, que en cierta medida han servido para el desarrollo de la economía ecuatoriana; pero la alta dependencia estatal hacia los ingresos petroleros, se ha constituido en un riesgo latente, debido a factores externos que inciden en los mercados internacionales.

Esto ha afectado en gran medida a la economía local, ejemplo de esto es lo que sucedió a finales del 2014, con la reducción del precio del petróleo observada desde inicios del segundo semestre, impactando directamente al equilibrio fiscal, al reducir los ingresos petroleros del sector público. Desde Julio 28 hasta el 10 de diciembre del 2014 el petróleo West Texas Intermédiate (WTI), de referencia para Ecuador, tuvo una reducción de $41 \%$ en su precio (de USD $\$ 101,67$ a UDS $\$ 59,65$ ), cerrando el 7 de marzo del 2016 en USD $\$ 37,90$ por barril, lo cual deja al crudo ecuatoriano alrededor de USD 20 por barril. Un escenario totalmente distinto al de junio del 2014 donde el precio llegó a su punto más alto de \$115 el barril (Maldonado \& Proaño, 2015). 


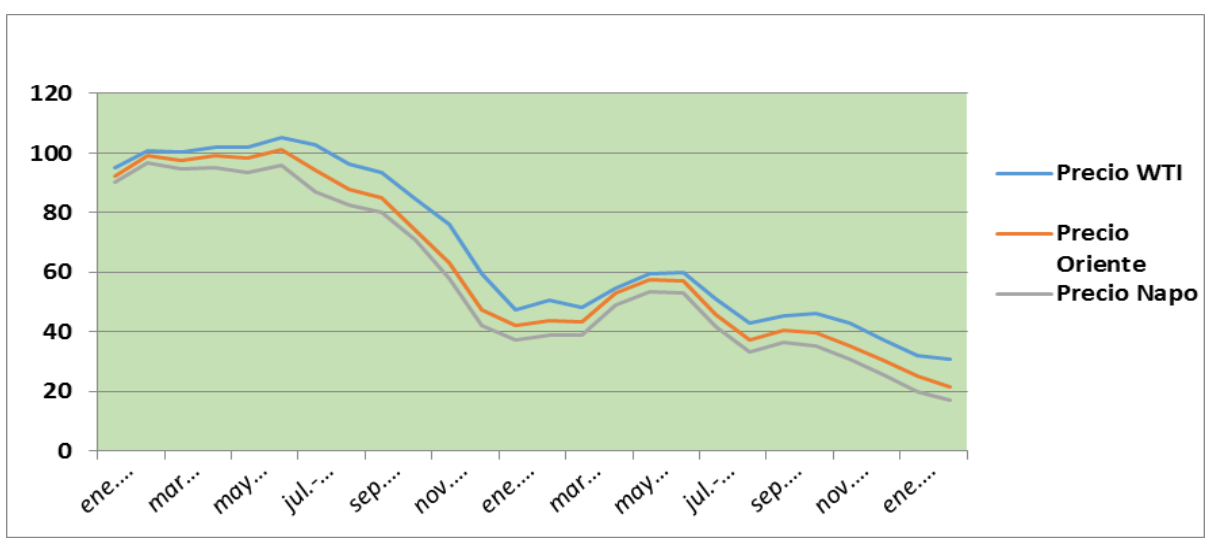

Figura 1. Precio Wti vs Oriente Y Napo 2014 - 2016.

Fuente: (Sistema Nacional de Información, 2016).

Ante este escenario, en el Ecuador de acuerdo Al Ministerio de Finanzas (2015), en el año 2015 el Presupuesto General del Estado sufrió dos ajustes; el primero en enero de USD $\$ 1420$ millones de dólares, y el segundo en el mes de agosto por UDS $\$ 800$ millones (USD $\$ 100$ millones en gasto corriente, y USD $\$ 700$ millones en gastos de inversión), con un recorte total de UDS $\$ 2220$ millones de dólares.

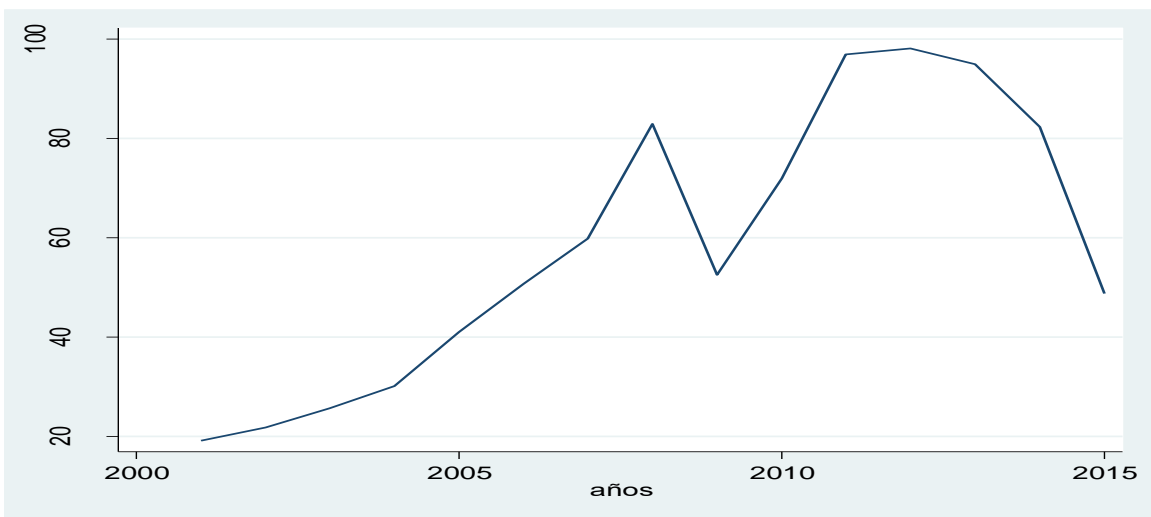

Figura 2. Variación de los precios del barril de petróleo desde del 2000-2015

Fuente: (Sistema Nacional de Información, 2016).

Como se puede observar en el grafico 2, en los últimos 15 años los precios del barril de petróleo han sufrido grandes variaciones, desde el año 2014 se presenta uno de los escenarios más complejos que le ha tocado confrontar económicamente al país y al gobierno. 


\section{METODOLOGÍA}

La presente investigación es de tipo cuantitativa y nos permite usar datos estadísticos, procesar información y analizar la interacción dinámica que pueda existir entre las variables objeto de estudio.

Las variables que se consideran para análisis econométrico fueron tomadas del estudio de Carrión, Kingman, \& Vargas (2002), quienes observaron que las variables que se consideran más explícitas para medir la dependencia petrolera del Ecuador son: el peso de la deuda externa en el PIB, y el precio del petróleo en el PIB, en las exportaciones y en el Presupuesto General del Estado.

Las variables objeto de estudio que se utilizan en el modelo econométrico son:

- Precio del barril de petróleo: precio promedio (Napo, Oriente, WTI). Variable independiente.

- Balanza Comercial: Variable dependiente.

- Presupuesto General del Estado: Variable dependiente.

Los datos estadísticos obtenidos fueron adquiridos de los boletines anuales desde año 2001 hasta el 2015 de la página web del Banco Central del Ecuador (https://www.bce.fin.ec/) y del Sistema Nacional de Información (sni.gob.ec/).

El objetivo central de la presente investigación es; Analizar las causas y efectos de las variaciones del precio del petróleo en dos principales variables de la economía Ecuatoriana: (Balanza comercial, Presupuesto General del Estado), a través de un modelo de regresión lineal Gujarati y Porter (2010).

Se define el modelo como "el estudio de la dependencia de una variable (variable dependiente) respecto de una o más variables (variables explicativas)", que permite observar la magnitud de cómo se vinculan las dos variables: una independiente $(X)$ y otra independiente $(\mathrm{Y})$.

El modelo teórico de regresión lineal simple es:

$$
Y=\beta 0+\beta 1 X+u
$$
Ecuación (1)

A continuación se plantean diversas regresiones lineales con el propósito de correlacionar y observar la dependencia de las principales variables macroeconómicas en el Ecuador, frente a los precios del petróleo en los mercados internacionales.

En el siguiente modelo se busca medir el grado de dependencia de la balanza comercial del Ecuador, ante el precio del barril del petróleo. Con los datos obtenidos de la tabla No. 2, se plantea la siguiente regresión lineal:

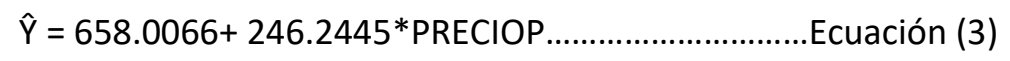


Tabla 2. Modelo de regresión lineal simple. Balanza comercial explicada por precio promedio del barril de petróleo.

\begin{tabular}{|c|c|c|c|c|c|c|}
\hline Source & SS & df & MS & Number of obs & $=$ & 15 \\
\hline & & & & $F(1,13)$ & $=$ & 121.18 \\
\hline Model & 666910652 & 1 & 666910652 & Prob> F & $=$ & 0.0000 \\
\hline \multirow[t]{2}{*}{ Residual } & 71542225.2 & 13 & 5503248.09 & R-squared & $=$ & 0.9031 \\
\hline & & & & Adj R-squared & $=$ & 0.8957 \\
\hline Total & 738452877 & 14 & 52746634.1 & Root MSE & $=$ & 2345.9 \\
\hline BComercial & Coef. & Std. Err. & $\mathrm{t}$ & $P>t$ & {$[95 \%$ Con } & Interval] \\
\hline PRECIOP & 246.2445 & 22.36878 & 11.01 & 0.000 & 197.9197 & 294.5693 \\
\hline _cons & 658.0066 & 1441.297 & 0.46 & 0.656 & -2455.726 & 3771.739 \\
\hline
\end{tabular}

Fuente: elaboración propia.

El modelo planteado indica que la balanza comercial en Ecuador aumenta en USD \$246.24 millones, por cada unidad que se incrementa en el precio del barril de petróleo. La dependencia de la Balanza Comercial ante el precio del barril de petróleo es altamente significativa, ya que al observar el valor de $\mathrm{P}=0.000(<0.05)$ con una confianza del $95 \%$ se deduce que la variable independiente PRECIOP sí explica e incide de forma directa y progresiva ante la variable dependiente $Y$ (B. comercial).

De la misma manera La R2 es una medida cuantitativa que nos permite observar que tan bien la variable independiente predice a la variable dependiente; en este modelo se obtuvo un R2= 0.903 , lo que significa que el precio del barril de petróleo define en un 90.31\% la variabilidad de la Balanza Comercial del Ecuador.

En la siguiente Tabla se vinculan dos variables al modelo; la variable balanza petrolera, frente a la variable presupuesto general del estado; con los datos obtenidos se propone el siguiente modelo:

$\hat{Y}=-8.953 .213+2.263 .139 * B$ Petrolera Ecuación (5)

Tabla 3:.Presupuesto General del Estado explicado por la balanza petrolera.

\begin{tabular}{|c|c|c|c|c|c|c|}
\hline Source & SS & df & MS & & Number of obs = & 15 \\
\hline & & & & & $F(1,13)$ & $=19.90$ \\
\hline Model & $1,08 \mathrm{E}+13$ & 1 & $1,08 \mathrm{E}+13$ & & Prob> F & $=0.0006$ \\
\hline Residual & 704125824 & 13 & 54163524.9 & & R-squared & $=0.6048$ \\
\hline & & & & & Adj R-squared & $=0.5744$ \\
\hline Total & $1,78 E+13$ & 14 & 127274560 & & Root MSE & $=7359.6$ \\
\hline PGE & Coef. & Std. Err. & $\mathrm{t}$ & $\mathrm{P}>\mathrm{t}$ & [95\% Conf. & Interval] \\
\hline BPetrolera & 2.263 .139 & .5073551 & 4.46 & 0.001 & 1.167 .065 & 3.359 .213 \\
\hline _cons & -8.953 .213 & 4.692 .935 & -0.19 & 0.852 & -11033.79 & 9.243 .149 \\
\hline
\end{tabular}

Fuente: elaboración propia. 
Tal como se observa en la tabla 3, el presupuesto general del estado, durante los 15 años observados ha sido totalmente dependiente de la balanza petrolera, es decir de los ingresos petroleros, ya que al observar el valor de $\mathrm{P}=0.000(<0.05)$ con una confianza del $95 \%$ se deduce que la variable independiente Balanza petrolera, incide considerablemente en la variable dependiente $Y$ (Presupuesto General del Estado). Adicional en este modelo se obtuvo un R2=0.6048 lo que significa que la balanza petrolera define en un $60.48 \%$ la variabilidad del PGE (Presupuesto General del Estado).

Con el objeto de corroborar lo mencionado en el modelo, se agrega la figura 3, que mide la relación entre los ingresos petroleros y el presupuesto general del estado.

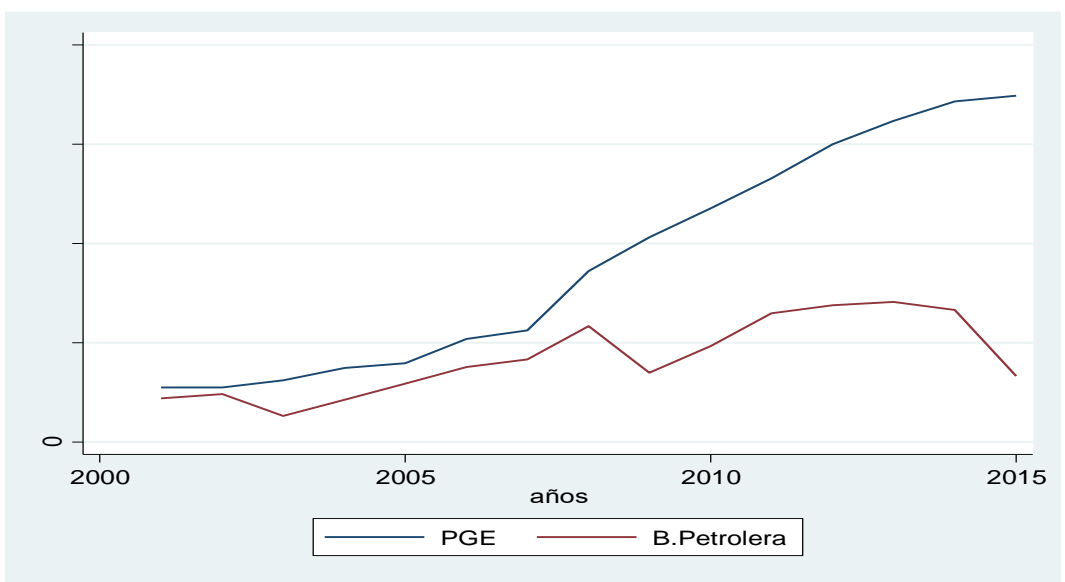

Por lo que podemos apreciar en la figura 3, donde se observa la relación entre el Presupuesto general del estado y los ingresos petroleros durante el periodo 2001-2015; la relación entre estas dos variables es alta entre los años 2001 al 2008, donde los ingresos presupuestados de nuestra economía están en función de lo que ocurra en el sector petrolero. Es a partir del año 2008 que se observa una cierta independencia del Presupuesto General del Estado ante los ingresos petroleros, y esto se debe entre otros factores, al fortalecimiento de los ingresos tributarios por parte del gobierno de la Revolución Ciudadana, pese a que durante esos años el precio del barril, se manejó con precios altos en los mercados internacionales, lo que fue un factor positivo para la liquidez del Ecuador. 


\section{CONCLUSIONES}

El escenario de la volatilidad de los precios del petróleo, depende de múltiples factores de carácter geopolíticos, económicos, sociales, inclusive ambientales; además que quienes marcan las reglas de juego son aquellos países productores que mantienen el liderazgo en cuanto a producción y comercialización, lo que desplaza el poder de negociación de los países que producen en menor escala, y que más aun poseen un petróleo de menor calidad como es el caso Ecuatoriano.

Los resultados obtenidos en las dos regresiones planteadas, indican que existe una evidente relación entre el precio del petróleo con las dos variables macroeconómicas analizadas: balanza comercial y presupuesto general del estado.

Se pudo observar la alta dependencia que el Ecuador tiene de los ingresos petroleros, de manera fuerte hasta el año 2008, de ahí hacia adelante en la economía ecuatoriana se observan otros factores económicos que han reducido la dependencia del petróleo. Sin embargo la inestabilidad que genera la variación constante de los precios de esta materia prima, aún sigue impactando en la economía del Ecuador, evidencia de ello es lo que sucedió entre los años 2014 - 2'016, donde se sintió una fuerte recesión económica, inclusive señalando el gobierno central que el fenómeno se dio por la caída de los precios del petróleo en los mercados internacionales.

El Ecuador debería minimizar su dependencia ante este producto escaso, y potenciar la llamada matriz productiva que se contempla en el objetivo 10 del Plan Nacional del Buen Vivir propuesto por SENPLADES (2013); el país debe apostar a la creatividad e innovación, al fortalecer emprendimientos basados en el conocimiento, o en desarrollo de tecnologías. 


\section{REFERENCIAS BIBLIOGRÁFICAS}

Agencia Internacional de Energia. (2012). World energy. World Energy Outlook, 43-46.

Arroyo, A., \& Cossío, F. (2015). Impacto fiscal de la volatilidad del precio del petróleo en América Latina y el Caribe, 88.

Banco Central del Ecuador. (2016). Relación ente el PIB y la Balanza comercial. Retrieved February 6, 2017, from https://www.bce.fin.ec/index.php/biblioteca-bce1

Barrañón, A. (2008). LA CRISIS MEXICANA DEL PETRÓLEO EN EL ESCENARIO DE PRECIOS ALTOS DEL PETRÓLEO. Razon Y Palabra, 13.

Blanchard, O., \& Gali, J. (2007). THE MACROECONOMIC EFFECTS OF OIL SHOCKS: WHY ARE THE 2000S SO DIFFERENT FROM THE 1970S? NATIONAL BUREAU OF ECONOMIC RESEARCH (Vol. XXXIII).

Carrión, F., Kingman, E., \& Vargas, G. (2002). Sobre bonanzas y dependencia Petróleo y enfermedad holandesa en el Ecuador. ICONOS, (13).

CORES. (2016). Informe estadístico anual 2015. CORES, 53(9), 1689-1699. http://doi.org/10.1017/CBO9781107415324.004

F.M.I. (2016). Perspectivas de la economía mundial, 1-7.

González, S., \& Hernández, E. (2016). Impactos indirectos de los precios del petróleo en el crecimiento económico colombiano. Lecturas de Economía, (84), 103-141.

Gujarati, D., \& Porter, D. (2010). Econometria. Statewide Agricultural Land Use Baseline 2015 (Vol. 1). http://doi.org/10.1017/CBO9781107415324.004

Hamilton, J. D. (2009). Causes and Consequences of the Oil Shock of 2007-08. Brookings Papers on Economic Activity, (1), 215-261. http://doi.org/10.1353/eca.0.0047

Hernández, L. (2015). EL MERCADO PETROLERO MUNDIAL Y LA CONSOLIDACIÓN DEL PROCESO DE LA INTEGRACIÓN ENERGÉTICA DE AMÉRICA LATINA Y EL CARIBE. Boletim Do Tempo Presente, (1919), 1-10.

Ito, K. (2010). The Impact of Oil Price Volatility on Macroeconomic Activity in Russia. Atlantic Review of Economics (2011-2013) (Vol. 9).

Kilian, L. (2008). The Economic Effects of Energy Price Shocks. Journal of Economic Literature, 46(4), 871-909. http://doi.org/10.1257/jel.46.4.871

López, A., \& Hilario, J. (2008). GEOPOLITICA del petróleo y crisis mundial. DYNA Revista de La Facultad de Minas Universidad Nacional de Colombia, 75(156), 1-8.

Lorca, J. M. (2015). El impacto de la crisis del petróleo de 1973 en el contexto económico español. Espacio, Tiempo Y Forma, 165-180.

Maldonado, F., \& Proaño, G. (2015). Perspectivas económicas 2015.

Marte, O., \& Villanueva, B. (2007). Los precios internacionales del petróleo, el pib real y los precios en la economía dominicana. Ciencia Y Sociedad, XXXII(2), 190-216.

Mateo, J. P., \& García, S. (2014). The Oil Sector in Ecuador. 2000-2010. Revista Latinoamericana de Economía, 45(177), p 113-139. 
Ministerio de Finanzas. (2015). RECORTE ADICIONAL DE US\$800 MILLONES EN PRESUPUESTO GENERAL DEL ESTADO 2015 EI (Vol. 1).

Senplades. (2013). Inicio - Plan Nacional 2013 - 2017. Retrieved February 16, 2017, from http://www.buenvivir.gob.ec/

Sistema Nacional de Información. (2016). Cotización - Sistema Nacional de Información. Retrieved February 6, 2017, from http://sni.gob.ec/cotizacion

Vargas, E. (2008). PETROLEO, BIOCOMBUSTIBLES E INFLACIÓN. Perspectivas, 21, 39-62. 